\title{
Craniopharyngioma Brain Invasion Forms
}

\section{Martha Lilia Tena-Suck ${ }^{1,2 *}$, Ma Elena Hernández-Campos', Alma Ortiz Plataa ${ }^{3}$, Carlos Sánchez- Garibay $^{1,2}$, Citlaltepetl Salinas Lara ${ }^{2}$ and Carlos Peñafiel ${ }^{2}$}

\author{
${ }^{1}$ Sección de Estudios de Posgrado e Investigación de la Escuela Superior de Medicina, Instituto Politécnico \\ Nacional, México \\ ${ }^{2}$ Departamento de Neuropatología, Instituto Nacional de Neurología y Neurocirugía "Manuel Velasco \\ Suárez", México \\ ${ }^{3}$ Laboratorio de Neuropatología Experimental, Departamento de Neuropatología, Instituto Nacional de \\ Neurología y Neurocirugía "Manuel Velasco Suárez", México
}

*Corresponding author: Martha Lilia Tena-Suck, MD, PhD, Sección de Estudios de Posgrado e Investigación de la Escuela Superior de Medicina, Instituto politécnico Nacional; Departamento de Neuropatología, Instituto Nacional de Neurología y Neurocirugía "Manuel Velasco Suárez", Av. Insurgentes Sur 3877 Col. La Joya, Tlalpan CP 14269, Ciudad de México, México, Tel: (52)-5556063822 ext. 2008, Fax: (52)-5554240808

\begin{abstract}
Background: Craniopharyngiomas constitute 2 to $4 \%$ of intracranial neoplasms. Although this tumors exhibit a benign histopathological pattern, recurrence is commonly reported by cerebrospinal fluid seeding and direct brain invasion. The aim of this study was to analyses and recognizes craniopharyngioma pathological features in relationship to brain invasion as a prognostic factor.
\end{abstract}

Methods: One hundred patients who were surgically treated for Craniopharyngiomas extending outside the sellar and suprasellar regions were evaluated. Clinical, pathological and immunohistochemistry profile correlations were done.

Results: The age of the patients varied from 15 to 87 years (mean $49.4 \mathrm{yrs}$ ); 41 (41\%) patients were males and 59 (59\%) were females. Brain invasion was found in 51 cases, recurrence occurred in 45 patients and 11 died. The follow-up was for $30.12 \pm 13.82$ months. The arrange mean of KI67-Li in tumoral nest cell invasion was $32.04 \pm 6.57$ vs. $16.68 \pm$ 4.0 for non tumoral invasion $(p=0.000)$. The intratumoral MVD-Li (CD34) mean range in invasive tumors was $4.84 \pm$ 1.06 vs. $4.88 \pm 1.01$ in non-invasive tumors $(p=0.462)$. The amount of MVD-li in peritumoral area was $17.98 \pm 4.67$ vs. $14.84 \pm 5.16$ of non peritumoral $(p=0.017)$. Higher Ki67-Li in neoplastic nest cells, were correlated with higher peritumoral MVD-Li ( $p=0.033)$, and E-Cadherin, $\beta$-tubulin, NFG and D2DR intratumoral positive immnunoexpression.
Conclusion: Invasion in craniopharyngioma is a common event that it is associated, to neuronal differentiation in tumoral cells and hypoxic chances. The neoplastic nest cells in the adjacent brain tissue showed a higher labelling index, higher peritumoral microvascular density, but not always it was associated to recurrence.

\section{Keywords}

Craniopharyngiomas, Invasion, Brain adjacent tumor

\section{Introduction}

Craniopharyngiomas (CPs) constitute approximately 2 to $4 \%$ of all primary intracranial tumours. Two types of Craniopharyngiomas are usually recognized; adamantinomatous (ACP) and papillary variant (PCP) [1]. $\mathrm{CP}$ commonly is histologically benign tumor clinically aggressive with high recurrence, frequently with local invasion and frequent recurrences as well as malignant transformation [1-3]. The histologically invasive findings were defined using the criteria by Weiner, et al. [4]. The presence of an isolated nest of tumor cells surrounded brain tissue, which was clearly distinct from the remainder of the tumor and described brain invasion as predictor factor in association with recurrence [4]. The way of brain invasion and adjacent tissue infiltration is rare and different shapes are observed histo- 
logically; e.g. nests, cords, and loose cells in the form of wet keratin, ghost cells and calcifications $[5,6]$. Understanding the growing or development of these features is not well known so far. A major challenge is finger-like tumor protrusions into the surrounding brain parenchyma, which makes gross total removal difficult to achieve and accounts for tumor relapse [7]. Tissue invasion and infiltration by brain tumors carriages a clinical trial, with destruction of structures leading to morbidity. Expectantly, be a guide to find new prognostic markers capable of estimating the risk of progression or recurrence. $\mathrm{CP}$ makes a tumor-specific cellular environment at the tumor-brain junction. Whether this helps the infiltrative growth pattern or is the consequence of an activated Wnt signaling pathway [7].

Recently have been observed high mutation rates of BRAF V600E in PCP and of CTNNB1 in ACP. These activating driver mutations are potential therapeutic targets $[8,9]$. Recent data indicates that both variants are defined by specific genetic alterations and influenced by distinct molecular pathways and origins [9].

The purpose of our current study was to delineate and understand the morphologic and immunophenotypic features in the different forms of brain invasion and from surrounding brain tissue in ACP as prognostic recurrence factors.

\section{Material and Methods}

From the neuropathology files at the Department of Neuropathology in the National Institute of Neurology and Neurosurgery at Mexico City, we included ACP that presented a clear pattern of invasion to adjacent brain tissue with the desire to identify the different patterns of infiltration and invasion. Clinical information and followup data were available, and all patients were managed with trans-sphenoidal or transcranial resections.

\section{Pathology}

Surgical and biopsy specimens from 100 patients with ACP were reviewed. Each specimen was classified according to World Health Organization (WHO) [1]. All biopsy samples were fixed overnight in $4 \%$ formalin and routinely processed into embedded paraffin. Sections were cut at $4 \mu \mathrm{m}$. All tumors analyzed in the study were obtained from the first surgery and were assigned to two categories according presence (group 1) or absence (group 2) of brain invasion.

The criteria for brain invasion including presence of cohesive cells of intermediate size, compactly arranged interdigitation as well as finger like, single cells, nests, cords in the form of wet keratin, ghost cells, and dystrophic calcification in brain parenchyma. Also, wet keratin, ghost cells and calcifications on the tumor boundaries were evaluated.

\section{Immunohistochemistry}

Immunohistological staining patterns employed the Dual Link Envision + detection system (DAKO Carpintery CA). Immunohistochemical studies were performed upon $5 \mu$ formalin-fixed, paraffin-embedded sections using antibodies directed against glial fibrillary acidic protein (GFAP) (DAKO, polyclonal; 1:4000), nestin (Spring Bioscience clone E-8750, 1:100), Vimentin (DAKO, clone Vim3B4 1:100), neurofilament protein (DAKO, clone 2F11, 1:75), Cytokeratin 7 (Santa Cruz, CA. 1:100), Cytokeratin 8 (DAKO, 1:100) E-cadherin (Zymed, clone 4A2C7; 1:2000), CD34 (DAKO, clone QBEnd10; 1:100), B-catenin (H-102) | SCBT - Santa Cruz Biotechnology; dilution 1:100), Dopamine D2 receptor (D2DR Santa Cruz CA, clonesc-5303, dilution 1:100), Dopamine Receptor 5 (D5DR, Santa Cruz CA, sc-376088, dilution 1:200) Interferon-Inducible factor (IFI-16, Santa Cruz CA, clonesc-802, 1:100), Hypoxic inducible factor alfa (HIF-1 $\alpha$, Santa Cruz CA, clone sc-4438 WB, dilution 1:75), Claudin 5 (and Ki67 (DAKO, clone MIB-1, 1:100). Standard immunostaining techniques as described by standard kit manufactures procedures, with appropriate positive and negative controls were used.

On immunohistochemically markers were scored in gliosis, epithelial cells, the stellate reticulum, cell ghosts, dystrophic calcifications and wet keratin, in the way of infiltrating into single cells, nests or in a mixed form component when feasible. If only one component was identified in the slide, then that component was evaluated exclusively. The differentiation of primary antibodies used were assessed on a semi quantitative scale: no staining (0); focal $<10 \%$ of the cells $(1+)$; $10-50 \%$ of cells or weak staining in $>50 \%$ of cells $(2+)$ and strong staining of $>50 \%$ of cells $(3+)$. MIB- 1 index (Ki-67) and microvascular density MVD (CD34) labelling index were obtained. Quantification of proliferating labeling index was determined by counting the number of immunostained nuclei of epithelial cells in five fields at 400X magnification. Microvessel density labeling index was also performed.

The microvascular density index (MVD-Li) expressed by CD34 antibody was defined according to procedures described by Vidal, et al. [10].

\section{Electron microscopy}

Statistical methods: For statistical analysis, we used the Mann-Whitney test, the paired t test coefficient of correlation and Spearman and Pearson correlation text. (Statistical analyses were performed with SPSS 20 software). Statistical significance was attributed to $p$ values less than $<0.05$. Patient and tumour characteristics were described with mean, ranges, and frequencies. All tests were 2 -sided with any $p$-value less than 0.05 considered statistically significant.

\section{Results}

The clinical characteristics were correlated with 
Table 1: Clinical characteristic of the patients with craniopharyngioma.

\begin{tabular}{|l|l|l|l|}
\hline & Brain invasión & Non invasión & P value \\
& $\mathbf{n = 5 1}$ & $\mathbf{n = 4 9}$ & \\
\hline Age & $50.25 \pm 13.82$ ys & $48.08 \pm 11.96$ ys & 0.361 \\
\hline Female & $24(53)$ & $35(69)$ & 0.037 \\
Male & $26(51)$ & $15(37)$ & 0.433 \\
\hline Time symptoms presentation & $11.08 \pm 4.39$ & $10.08 \pm 3.69$ & 0.220 \\
\hline Recurrence & $26(51)$ & $19(39)$ & 0.042 \\
\hline Panhipopitituarism & $23(45)$ & $13(27)$ & 0.335 \\
\hline Insipidus diabetes & $24(47)$ & $20(41)$ & 0.718 \\
\hline Total exeresis & $33(65)$ & $18(37)$ & $19(39)$ \\
\hline Partial exeresis & $30(59)$ & $4(9)$ & 0.038 \\
\hline Death & $7(14)$ & $34.59 \pm 8.48 \mathrm{mo}$ & 0.374 \\
\hline Follow-up & $30.12 \pm 13.82 \mathrm{mo}$ & 0.001 \\
\hline Tumor size & $36.35 \pm 6.36$ & $34.69 \pm 5.91$ & 0.348 \\
\hline
\end{tabular}

cc: Coefficient of correlation of Sperman and Pearson. Age, tumor size and follow-up were calculated by mean and standard derivation.

Table 2: Histopathology features associated to tumor in normal brain adjacent tissue (brain invasion).

\begin{tabular}{|c|c|c|c|}
\hline & $\begin{array}{l}\text { Brain invasión } \\
n=51(\%)\end{array}$ & $\begin{array}{l}\text { Non invasión } \\
n=49(\%)\end{array}$ & $P$ value \\
\hline Wet keratin & $33(67)$ & $28(57)$ & 0.284 \\
\hline Ghost cells & $30(59)$ & $26(53)$ & 0.352 \\
\hline Dystrophic Calcificacion & $43(84)$ & $40(82)$ & 0.165 \\
\hline Glomerulus & $26(51)$ & $26(53)$ & 0.497 \\
\hline Gliosis & $33(67)$ & $28(57)$ & 0.284 \\
\hline Rosenthal Fibres & $36(51)$ & $29(59)$ & 0.191 \\
\hline Macrophages & $23(45)$ & $21(43)$ & $\begin{array}{l}0.513 \\
\text { cc0.017 }\end{array}$ \\
\hline Inflammation & $22(43)$ & $20(41)$ & 0.355 \\
\hline Chef colesterol & $14(27)$ & $13(27)$ & $\begin{array}{l}0.549 \\
\text { cc0.010 }\end{array}$ \\
\hline
\end{tabular}

cc: Coefficient of correlation of Sperman and Pearson.

brain invasion and the results are seen in Table 1 . The age of patients varied from 15 to 87 years (mean 49.4 yrs); 41 (41\%) were males, and 59 (59\%) were females. Tumour size varied from 23 to $49 \mathrm{~mm}$ (mean 35.54 $\mathrm{mm})$.

Relevant histological features according with brain invasion are summarized in Table 2 . We observed that the invasion to brain parenchyma is more common than has been reported. The way of invasion may be in the form of interdigitation as well as finger like (Figure 1a), single cells (Figure 1b), nests or cords (Figure 1c, Figure $1 d$ and Figure 1e), dystrophic calcification (Figure 1f), ghost cells (Figure 1g), and by a mixed pattern where there are one or two elements together (Figure $1 \mathrm{~h}$ ).

The way of tissue invasion can be quite varied, but it is common that single cells grow to form nests and cords accompanied by calcification. Dystrophic calcifi- cations were observed isolated or usually the ghost's cells and wet keratin calcify when they do not find an adequate stream (Figure $1 \mathrm{~g}$ and Figure 1h). Therefore, the hypothesis that ghost cells are also shoved or spirited out of the tumour and that those unable to find an appropriate way to survive are calcified (Figure $1 \mathrm{~g}$ ). Formation of a gliotic rim or scare around the lesion was detected in all patients, although in a varying degree.

The results of immunostaining are summarized in Table 3. GFAP immunoreactivity was seen in the cell bodies of reactive astrocytes but also within fibrillary processes. Glass is usually found around the external epithelium and especially when there is a rupture of the tumour; higher glass was found around the epithelial nests and in mixed pattern and none was associated to invasive or poor prognosis (Figure 1f). When GFAP was not detectable in the respective tumor cells, areas resembling fibrosis degeneration within CPs tissue dis- 

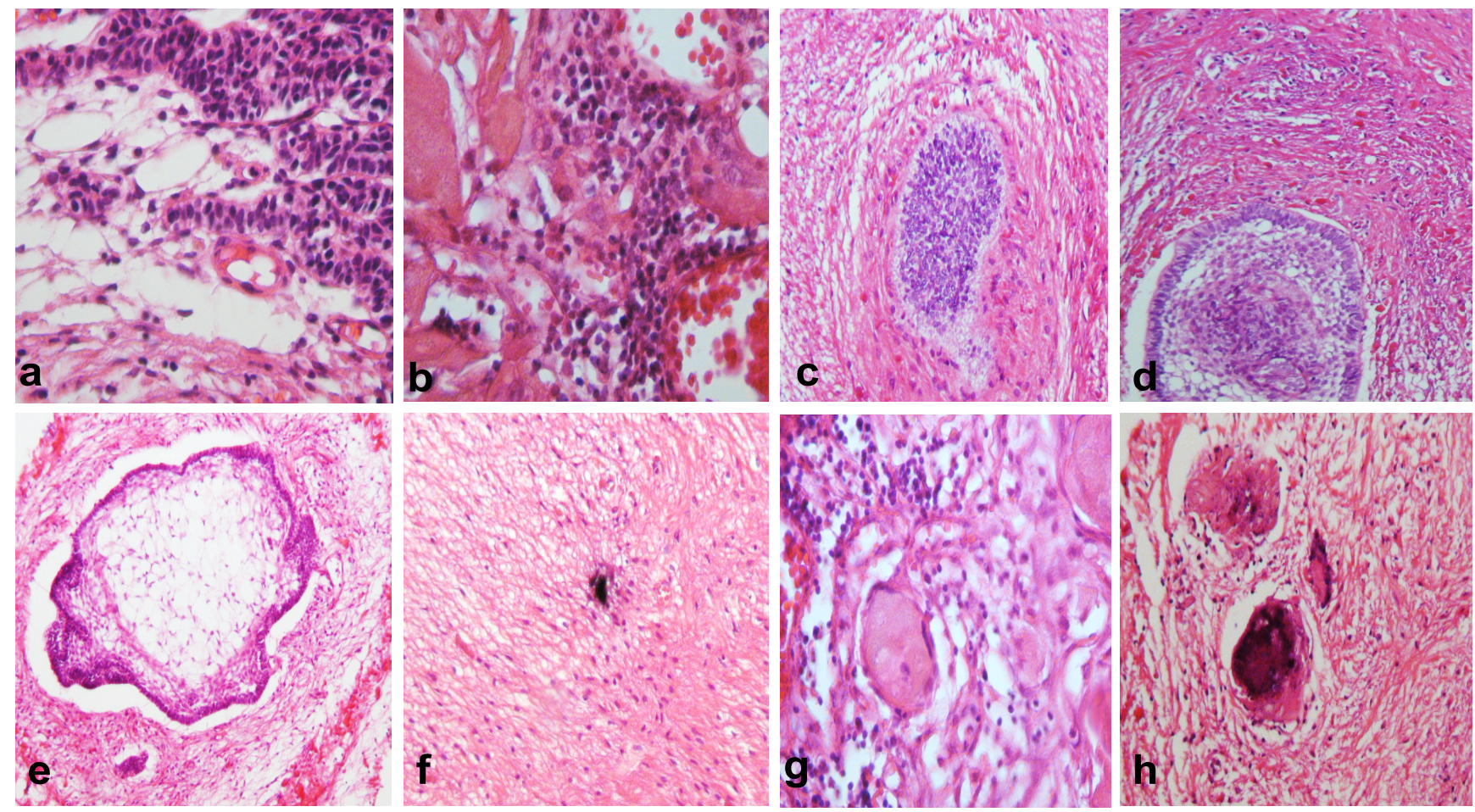

Figure 1: Histology of the craniopharyngioma brain invasion features: (a) Interdigitations as well as finger like, (b) Single immature small neoplastic cells (c and d), formation of irregular nests. (e) The nests are of different sizes and shapes with proliferation of small immature cells that grow and showed a larger nest that formed the dashing stellate reticulum. (f) Dystrophic calcification in middle of brain parenchyma (H\&E x200). (g) Ghost cells and isolated immature small cells resembling lymphocytes, and in the mixed pattern. (h) Calcification in absence of an adequate stroma, gliosis and Rosenthal fibres (H\&E x400).
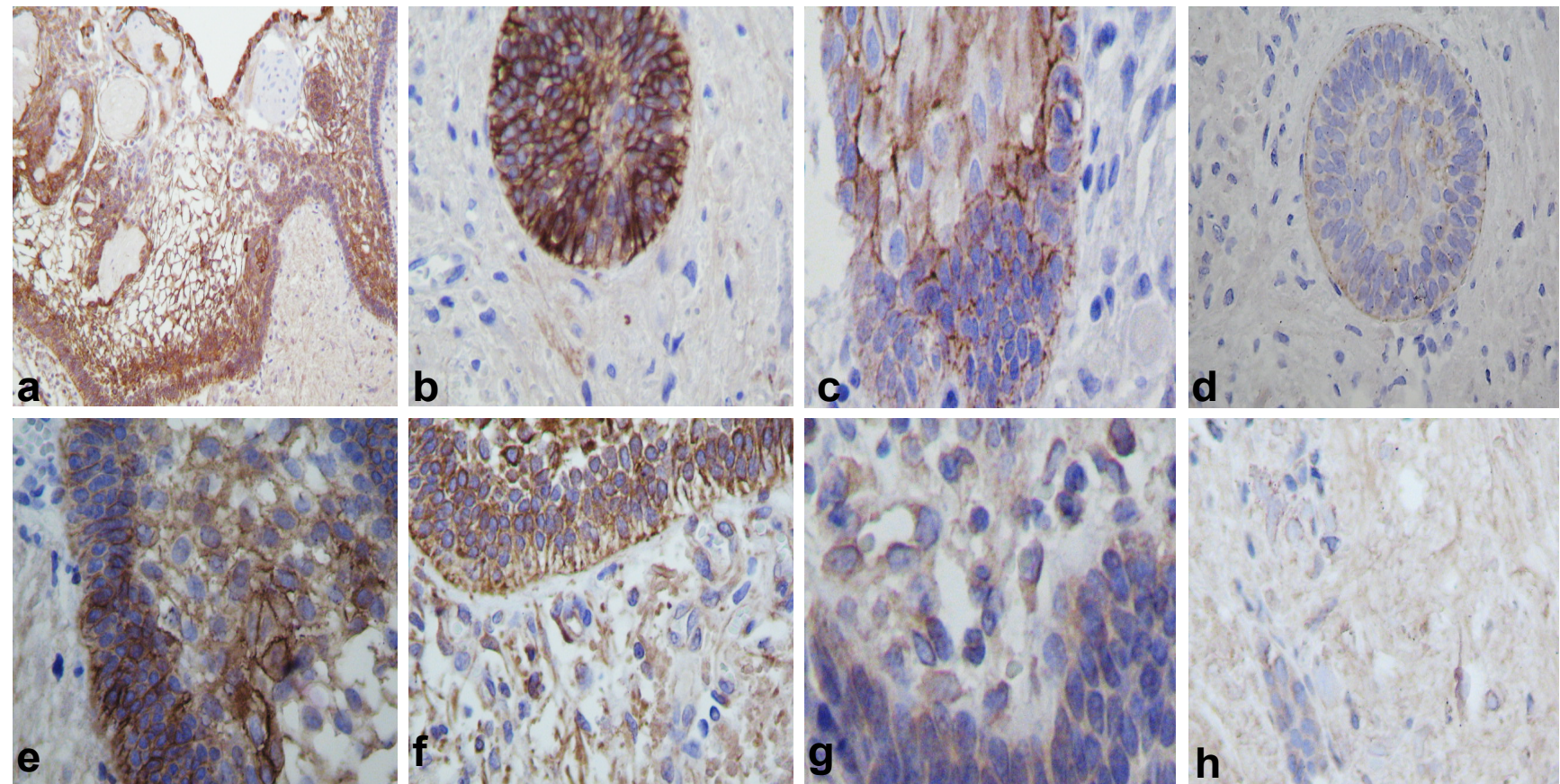

Figure 2: Immunohistochemistry: (a) Strong keratin 7 immunoexpression in external epithelium and weak immunoexpression in stellate reticulum, (b) Showed keratin 7 in nest infiltration. (c) Laminin was immunopositive in the basal membrane of the external epithelium. (d) Immunoreaction of nests in brain infiltration. (e) Neurofilament positive reaction in the external epithelium showed branching in the stellate reticulum. (f) Positive immunoreaction of the nest in brain parenchyma and in some isolated epithelial cells. (g) $\beta$-tubulin immunoreaction in the tumoral external epithelium. (h) Immunoreaction of the nest form in brain invasion (IHQ stain x400).

played strong GFAP expression and therefore may represent.
These external epithelial cells and temporal nests were positive for keratin 7 (Figure 2a and Figure 2b) and keratin 8 negative. In larger nests the expression 
Table 3: Immunohistochemistry results, according immunoexpression in tumoral cells and brain invasion.

\begin{tabular}{|c|c|c|c|}
\hline & $\begin{array}{l}\text { Brain invasion } \\
\mathrm{n}=51(\%)\end{array}$ & $\begin{array}{l}\text { Non invasion } \\
n=49(\%)\end{array}$ & $P$ value \\
\hline Ki67 external epithelium & $16.71 \pm 3.60$ & $16.51 \pm 3.75$ & 0.100 \\
\hline Ki67 reticulum stellate & $25.12 \pm 9.02$ & $26.12 \pm 9.03$ & 0.251 \\
\hline Ki67 nest invasión & $32.04 \pm 6.57$ & $16.68 \pm 4.0$ & 0.000 \\
\hline MVD-li (CD34) intratumoral & $4.84 \pm 1.06$ & $4.88 \pm 1.01$ & 0.462 \\
\hline MVD-Li (CD34) peritumoral & $17.98 \pm 4.67$ & $14.84 \pm 5.16$ & 0.017 \\
\hline Keratin 7 tumoral & $41(80)$ & $40(82)$ & $\begin{array}{l}0.539 \\
\text { cc. } 016\end{array}$ \\
\hline Keratin 7 in nest/invasion & $3(6)$ & $2(4)$ & 0.000 \\
\hline Keratin 8 tumoral & $51(100)$ & 0 & 0.000 \\
\hline Keratin 8 in nest/invasion & $51(100)$ & 0 & 0.000 \\
\hline E-cadherin intratumoral & $51(100)$ & 0 & 0.000 \\
\hline E-Cadherin-in invasión & $51(100)$ & 0 & 0.000 \\
\hline IFI-16 intratumoral & $29(57)$ & $36(73)$ & 0.063 \\
\hline IFI-16 invasion & $51(100)$ & 0 & 0.000 \\
\hline HIF1 $\alpha$ tumoral & $29(57)$ & $36(73)$ & 0.063 \\
\hline HIF1 $\alpha$ intratumoral & $51(100)$ & 0 & 0.000 \\
\hline Tubulin intratumoral & $10(20)$ & $10(20)$ & $\begin{array}{l}0.559 \\
\operatorname{cc} 0.010\end{array}$ \\
\hline Tubulin invasión & $51(100)$ & 0 & 0.000 \\
\hline D2DR intratumoral & $10(20)$ & $10(20)$ & $\begin{array}{l}0.559 \\
\operatorname{cc} 0.010\end{array}$ \\
\hline D2DR invasión & $10(20)$ & $10(20)$ & $\begin{array}{l}0.559 \\
\text { cc. } 010\end{array}$ \\
\hline NGF intratumoral & $51(100)$ & 0 & 0.000 \\
\hline NGF invasión & $10(20)$ & $10(20)$ & $\begin{array}{l}0.559 \\
\operatorname{cc} 0.010\end{array}$ \\
\hline Neurofilaments intratumoral & $51(100)$ & 0 & 0.000 \\
\hline Neurofilaments invasión & $10(20)$ & $10(20)$ & $\begin{array}{l}0.559 \\
\operatorname{cc} 0.010\end{array}$ \\
\hline Vimentin intratumoral & $10(20)$ & $10(20)$ & $\begin{array}{l}0.559 \\
\operatorname{cc} 0.010\end{array}$ \\
\hline Vimentin invasión & $51(100)$ & 0 & 0.000 \\
\hline Nestin intratumoral & $20(20)$ & 10 & 0.005 \\
\hline Nestin invasión & $51(100)$ & 0 & 0.000 \\
\hline EVGF intratumoral & $51(100)$ & 0 & 0.000 \\
\hline EVGF invasión & $10(20)$ & $10(20)$ & $\begin{array}{l}0.559 \\
\text { cc0.010 }\end{array}$ \\
\hline
\end{tabular}

cc: Coefficient of correlation of Sperman and Pearson. NGF: Neural grow factor, (D2DR.- Dopamine D2 receptor Interferon- IFI16 Inducible factor, D5DR Dopamina Receptor 5. IFI-16: Gamma-interferon-inducible protein Ifi-16 (also known as interferoninducible) myeloid differentiation transcriptional activator, HIF-1a HIF1A; hypoxia inducible factor 1, alpha subunit.

was higher for laminin (Figure $2 \mathrm{c}$ and Figure 2d), neurofilament (Figure 2e and Figure 2f) and $\beta$-tubulin (Figure $2 \mathrm{~g}$ and Figure $2 \mathrm{~h}$ ). NFG were positive in some cells of the external epithelium, in the stroma or in the stellate reticulum. Intensely positive neurofilament was observed in single cells and loose perivascular neoplastic cells (Figure 3a), also in areas where stream was more hyper cellular or dense (Figure 3b).

E-Cadherin, laminin, neurofilament and NFG are separated by a thin epithelial basement membrane, that when it is reached and broke down, proliferation of immature cells keratin negatives surrounded by larger vessels, as well as gliosis is observed. 

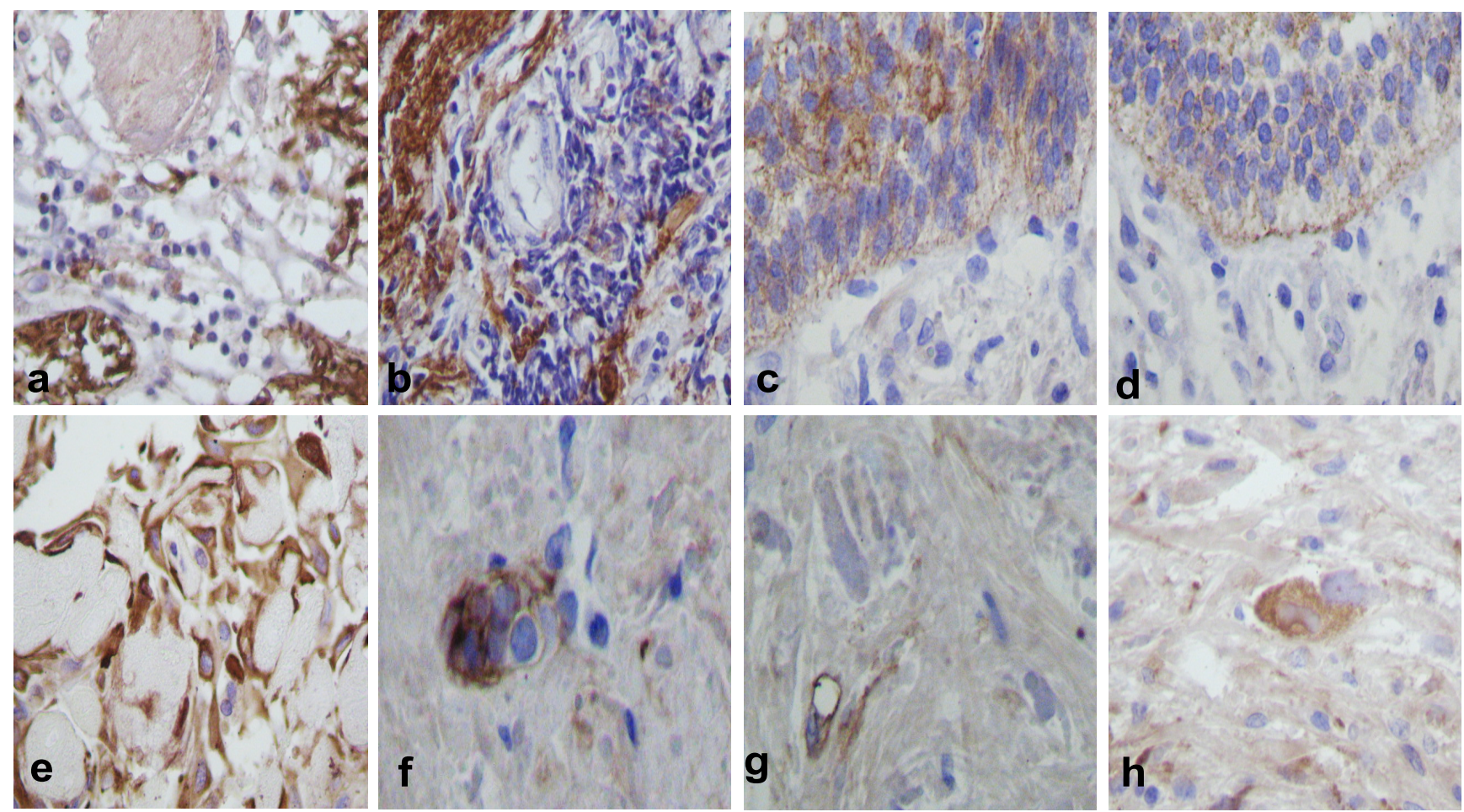

Figure 3: Immunohistochemistry: (a) Neurofilament positivity was observed also in cytoplasm of small immature cells, as well as in formation of thick filaments around them. (b) Likewise, the neurofilament was thicker and infiltrating into the stroma with increased cellularity. (c) E-Cadherin positive strong reaction in peripheral location, stroma and in external epithelium forming the basal membrane. (d) Aberrant or abnormal $\beta$-Catenin expression in the nests. (e) D2DR positive cells in the outer of dystrophic calcifications and ghost cells. (f) Positive isolated D2DR positive cells within the tissue, but negative in the brain parenchyma. ( $g$ and $h$ ) Few and isolated large cells in brain parenchyma with neuronal differentiation positive to D2DR were observed (IHQ stain x400).
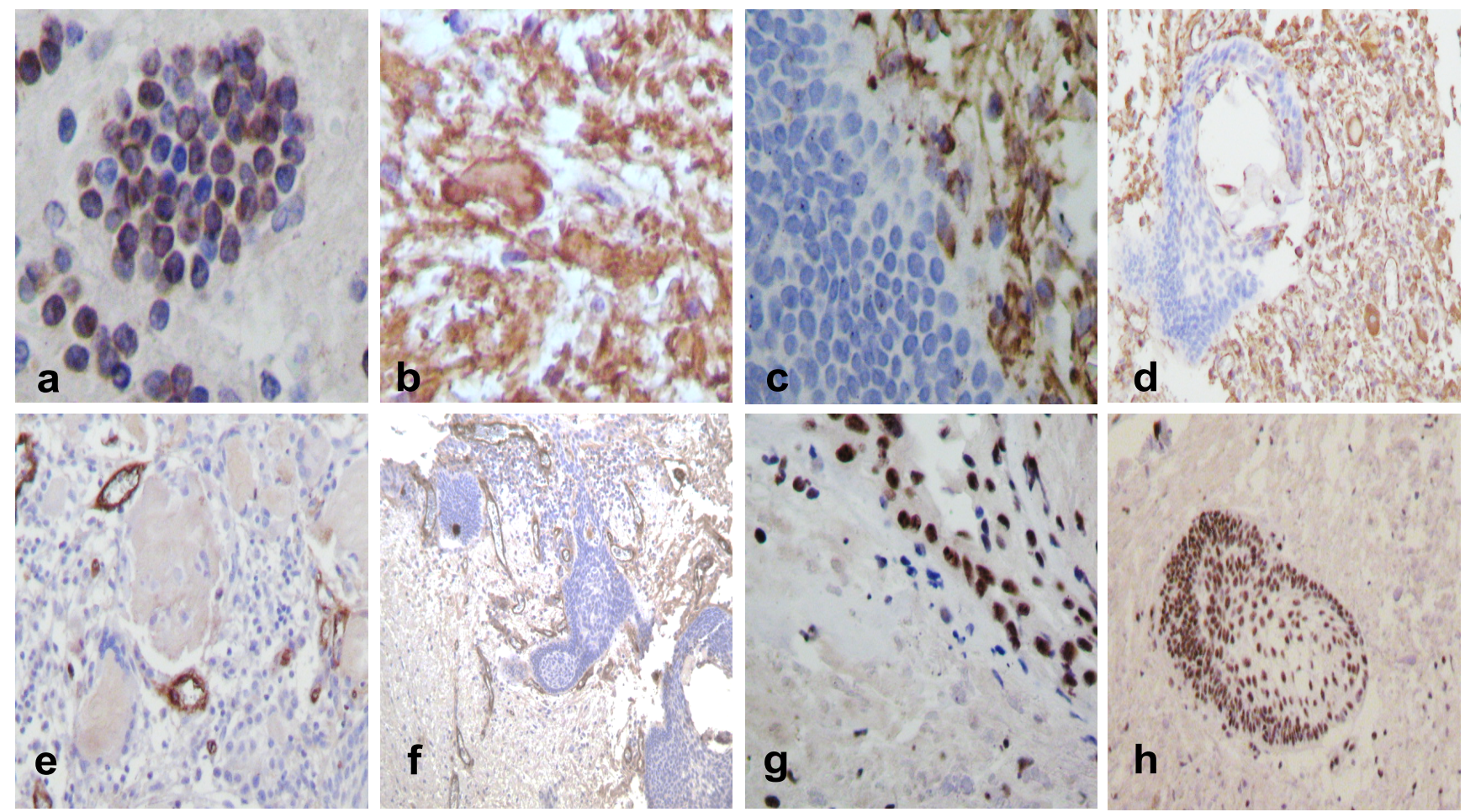

Figure 4: Immunohistochemical staining patterns of ACP using an antibody directed to IFI-16: (a) Positive cells in nest forming. (b) GFAP positive immunoreaction was strong in astrocytes and in the peripheral region of Rosenthal fibres. (c) Vimentin positive reaction was in peritumoral gliosis and in few isolated invading cells. (d) Vimentin was also positive as well in stroma differentiation in nest presenting. (e) Endothelial cell CD34 positive insides of the tumour. (f) Proliferative vessels on peritumoral area. (g) Ki-67 nuclear positive tumour cells, (h) and higher nuclear positive reaction in invasion nests. 
E-Cadherin was positive immunoreaction in peripheral location, external epithelium (Figure 3c), in nests and in arrays. Beta catenin was positive immunoreaction in nest, and in arrays (Figure $3 \mathrm{~d}$ ) and in isolate cells. However positive D2DR was observed on the outer of dystrophic calcifications, ghost cells (Figure 3e), in small nests (Figure $3 \mathrm{f}$ ) and within the tissue but were absent in the brain parenchyma. Large intensely positive cells suggest that neurons in case of being trapped in the neoplastic process reached cell differentiation and mature neurons that were strongly positive for D2DR (Figure $3 g$ and Figure 3h), D5DR and synaptophysin were negative in all cases.

IFI-16 and HIF-1 $\alpha$ were positive in small immature cells that were beginning to build nests and isolated cells. It was intensely positive in both the nucleus and strengthened toward the basement membrane (Figure 4a).

PGAF had strong immunoreaction in gliosis and in the peripheral of Rosenthal Fibres (Figure 4b). Vimentin was also positive in peritumoral gliosis, in some small cells, in spreading or expanding tumoral cells (Figure $4 c$ ), in the nests with vimentin positive gliosis, as well as in the process of differentiation of the nest and stroma (Figure 4d).

MVD-Li was higher around the tumour, on the external epithelium and in the brain, parenchyma affected by the tumor, especially in the stellate reticulum (Figure 4 e). In case of invasion MVD-Li was higher around the nests and in the mixed pattern hyper cellularity was observed if this last condition had calcifications (Figure 4f). The CD34 intratumoral-li invasion was $4.84 \pm 1.06$ vs. non-invasion $4.88 \pm 1.01(p=0.462)$. The MVD in peritumoral area was $17.98 \pm 4.67$ vs. non-invasion $14.84 \pm$ $5.16(p=0.017)$.

In relation to the Ki-67li, we observed higher scores when the stellate reticulum was solid, hypercellular (Figure 4g) and if the external epithelium showed rupture of the basement membrane and infiltrating nests (Figure 4h). Curiously we also observed increased $\mathrm{Ki}-67$ proliferation when neurofilament and $\beta$-tubulin expression were present on tumour stromal invasion.

The range of Ki67-Li on invaded external epithelium was $16.71 \pm 3.60 \%$ vs. $16.51 \pm 3.75 \%(p=0.100)$ in noninvasion; stellate reticulum Ki-67-Li invasion was $25.12 \pm$ 9.025 vs. $26.12 \pm 9.03 \%(p=0.251)$ in non-invasion and finally in nest invasion tumoral cells was $32.04 \pm 6.57$ vs. $16.68 \pm 4.0(p=0.000)$ non-invasion. Higher Ki67-Li in nests of neoplastic cells was correlated with CD34Li peritumoral cells $(p=0.033)$, endocrine disturbances $(p=0.049)$, age $(p=0.050)$, ghost cells $(p=0.010)$, dystrophic calcification ( $p=0.001)$, and E-Cadherin, $\beta$-tubulin, EVGF, laminin, NFG and D2DR intratumoral immnunoexpression ( $p=0.000$ respectively). Recurrence was correlated with brain invasion.
The results of electron microscopy are observed in semifinal sections as the external epithelium shows loss of the intracellular junctions in the presence of a dense material (Figure 5a), and the exit of the epithelial cells that slip easily through this material (Figure $5 b$ ).

\section{Discussion}

This study provides the first analysis of the expression of a large panel of tight junction proteins in CPs and boundary using immunocytochemistry. We demonstrate that CLDN-1, CLDN-5, e-Catherine and $\beta$-catenin were differently expressed in this tumor, and their localization in different histological findings suggests hypotheses on the histogenesis of these tumors. They play an important role in cell polarity and cell adhesion as well as in maintaining paracellular barrier functions [11]. Low CLDN1 expression levels correlate with an invasive $\mathrm{CP}$ growth pattern and may serve as an affecting cell mobility and tumor invasiveness [12]. Furthermore, we showed that expression of several other tight junction proteins is altered in human ACPs, confirmed morphology by electron microscopy and could be explain the brain invasion.

Tumor cell migration in ACP has been linked to cells with activated Wnt signalling pathway characterized by nuclear accumulation or dysregulation of $\beta$-catenin, as well as CLDN expression [8,9,13].

This way of invasion creates a tumor-specific cellular environment at the tumor-brain junction. Whether this facilitates the characteristic infiltrative growth pattern or is the consequence of an activated Wnt signalling pathway $[8,9]$. This hypothesis was widely discussed in the study of Burghaus $S$, et al. [7].

CP boundaries showed some interdigitating tumor cells in the surrounding gliotic layer adjacent to the CP $[7,9,10]$, but their irregular digital brain infiltration makes any complete surgical resection difficult to obtain. It is frequent that this tumor has very few vessels in epithelium and in the stellate reticulum, however the number of vessels increase in relation to cell dissemination, brain invasion with improved inflammatory response in the surrounding brain tissue $[1,10]$. Ultrastructural results suggested focal pathological disturbances and an altered microenvironment surrounding the tumor-brain junction, including damaging of the extracellular matrix (ECM) [14], and oil machinery fluid (OMF) liberation in the extracellular environment. When the OMF exits outward, either through pressure, rupture, opening of the epithelium, necrosis, or edema, etc, it produces a side effect that may be toxic to adjacent brain tissue [14]. Total removal of CF is burdened by high morbidity.

Little is known about the characteristics of Oil Machinery Fluid. The presence of $\beta$-thymosins in the OMF confirmed the secretion of these proteins in the extracellular environment [15]. These peptides and galectin-4 contributes with significant information to the de- 

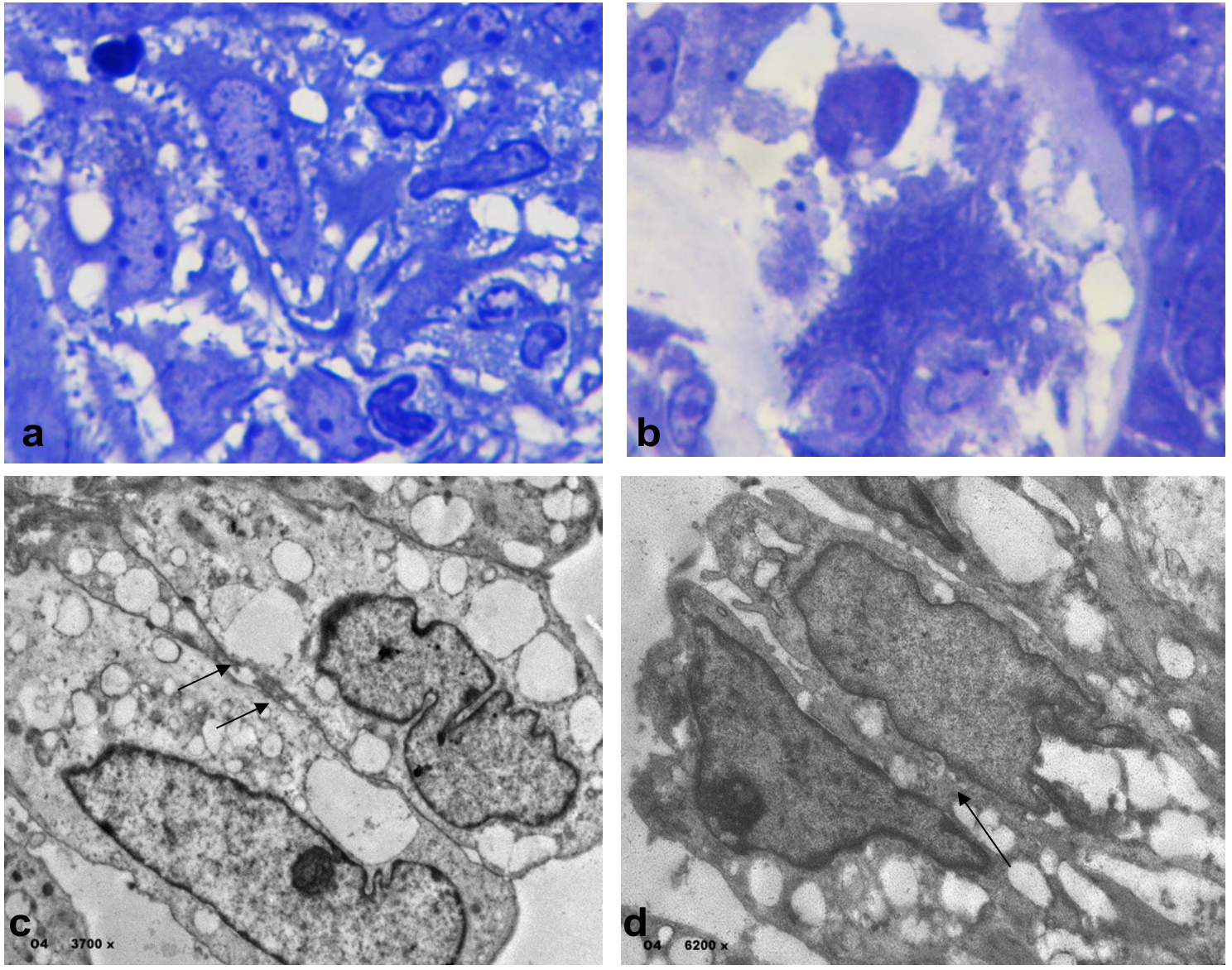

Figure 5: Electron microscopy analysis observed in semifinal sections as the external epithelium shows loss of the intracellular junctions in the presence of a dense material in (a), and the exit of the epithelial cells that slip easily through this material in (b) (toluidine blue stain $\times 100$ ). Epithelial cells showed cytoplasm's lipid droplets and intercellular interconnections abnormalities in (c) (4x 3700), and in (d) observed also lipid droplets in nucleus and lacking of nucleolus (4x 6200$)$. The arrows show abnormal intercellular corrections, open desmosomes.

lay in recurrence independently of surgical status [16], could be strictly involved in both tumor evolution and cyst progress and growth [14-16]. However, an intense inflammatory response, formation of granulomas has been observed in association with the rupture of the cystic structures [17].

CPs are, still, locally invasive and generate adherent interfaces with the surrounding brain parenchyma. Previous studies have shown the tumor microenvironment is characterized by a local abundance of leukocytes and elevated levels of pro-inflammatory cytokines that are supposed to be responsible, at least in part, for the local invasion with the surrounding brain parenchyma [16].

The accumulation of the OMF in adjacent brain tissue must be irritant and toxic [14], some remain quiescent before and after surgical decompression acute chemical meningitis has been caused by leakage and secondary irritation from the $\mathrm{CP}$ cyst different components [17]. Chronic degenerative or regressive or scene changes were also described in the surrounding brain tissue [18].

Burghaus S, et al. [7], suggested that ACP induce a tumor-specific cellular environment at the brain invasion border. This includes the occurrence of peculiar cell population of presumably astroglia precursors in close relation to the characteristic finger-like protrusions of ACP [7]. It also suggested that specific tumor and brain interaction effect of extracellular matrix (ECM) modulation and might have an influence on the growth pattern [7]. Our data showed that modulation of pre-existent vasculature can contribute to malignant progression and induction of sprouting angiogenesis. Continuing intravascular tumor expansion led to disruption of the blood-brain-barrier and overflow of cells that progressed along the vessels $[7,10]$. Our results showed a higher MVD in recurrence tumors vs. non-recurrent tumors and in rounding the nest [10].

Migration and invasion of epithelial cells are prevented by the formation of TJs, which are responsible for cell polarity and cell-cell adhesion within epithelial structures $[11,19]$. Immunoreactivity is restricted to cell groups located next to areas with extensive regressive changes (eg: calcifications) as well as surrounding whorl-like arrays, as well as diminished membranous and prominent cytoplasmic CLDN1 staining, correlates with the invasive potential of the certain cases [12]. Low CLDN1 expression levels correlate with an invasive CP growth pattern and may serve as a prognostic marker 
[12]. With these works we can better understand how the rupture of the cystic structures and the exit of the Oil Machinery Fluid contribute to the dissemination of the cells towards the cerebral parenchyma.

Burgess, et al. [7], uncovered a peculiar cell population, offering a glial phenotype with a distinct expression of both the embryo and the adult transcript of MAP2 and nestin. These cells occurred, especially near $A C P$, could represent primary adult glial cells, reactive astrocytes building a gliotic border, especially projecting along the finger-like protrusions [7]. In this study the co-expression of GFAP suggests their astrocytic origin, whereas localization is compatible with an immature developmental stage. Nesting is an established marker for stem/progenitor cells [7]. Furthermore, expression of various integrin subunits supports an interaction between CP tumor cells and ECM to provide adhesiveness, a precondition for tumor cell invasion [7]. The development of surrounding gliosis is well known for many types of tumors and is thought to be the responsible of the brain parenchyma hypoxia and inflammation as well as a presence of OMF outside of the cyst structures $[12,19]$, a supposition that must be established in further studies. When by surgery or by the effect of the same tumor the OMF comes out to the outside can be a big problem to the surgeon because during the same surgical process it can be disseminated even more. CP characterize a challenge for cure since of the adherence to the nearby nervous tissue and the high risk of hypothalamic damage during surgery; on the other hand, a partial removal enhances the risk of relapses.

The role of the ECM in tumoral genesis includes effects on epithelial polarity and angiogenesis [19]. The stroma and epithelial cells together regulate the expression and remodelling of the ECM [19]. The basement membrane separates the epithelial and endothelial cells from the stromal components [12]. However, the basal epithelium, myoepithelial cells and fibroblasts express the major components of the basement membrane (collagen $\mathrm{VI}$, laminin, entactin, and heparan-sulphate proteoglycans) in a tissue specific manner $[11,12]$. Higher micro vessel density values have been observed in recurrence and in association of cyst structure rupture and secondary hypoxic changes [17]. CPs are accompanied by a specialized stroma which is characterized by modifications in the non-epithelial cell types that secret extracellular matrix (ECM) proteins and growth factors $[12,19,20]$.

Several studies on the prognostic value of the immunoreactivity of the tumour proliferation marker MIB-1 have provided contradictory data [1], suggesting differences between recurrent and non-recurrent lesions. Furthermore, Weiner $\mathrm{H}$, et al. [4], found that the presence of one or more mitotic figures within the tumor not correlated with subsequent recurrence. In addition, recurrence not always has been correlated to brain in- vasion and in this study, we found to be associated to higher Ki67-li ranges in stellate reticulum cells and in whorl-like arrays formation and neuronal differentiation in invasive tissue and not in the tumoral external epithelium [1,4]. Furthermore, interdigitations of cells grow into cyst structures and then develop the stroma. The nests displayed a greater proliferation unspeakable in the tumor $[7,12]$.

Neural differentiation in CP is not well known, may be is due in association and activation of Wnt-signaling which serves as a promoter of the epithelial migration by regulating target molecules [8,9], or simply is an independent factor, or stem cells differentiation $[19,20]$. The authors reported in the nests observed in the brain adjacent tissue expressed laminin, D2DR, neurofilaments and $\beta$-tubulin and HIF-1, HIF-1, $\beta$-catenin and a higher MVD rounding those structures. This data is an important basis for further experimental strategies addressing molecular signalling pathways of epithelial tumor formation and infiltration into the CNS. Likewise, neural differentiation is a bit longer daring and risky, because we don't have genetic vs. molecular markers that could explain this event, neuronal differentiation in both the tumor and in invasion form is associated with poor prognosis [21] or it is a simply mechanism of cellular adaptation or cellular plasticity, Though, further studies are needed.

Evidence from mouse models suggests a key role for these clusters in tumor initiation progression and brain invasion is activating by CTNNB1 mutations $[9,21]$. The authors observed E-cadherin loss expression in a nest in recurrence tumors was non-recurring in association with aberrant B-catenin expression [12].

Tissue invasion and infiltration by brain tumours poses a clinical challenge, with destruction of brain structures or adjacent tissue and the failure to separate tumour and normal tissue warning the ability to perform a complete surgical resection required for cure.

Therefore, we suggest that the rupture of the outer epithelial recent evidence points towards a role in tumor progression and invasiveness in the brain segment membrane is one of the most important factors that may be in direct relation to invasion [22]. Hypothalamic changes, pathological or treatment induced, have major influence on prognosis in $\mathrm{CP}$ patients principally because of consequent hypothalamic obesity. Current insight in molecular genetics, treatment strategies, risk factors and outcomes associated with novel therapeutic perspectives [22].

\section{Conclusion}

Probably the way of invasion is in close relationship to the time of evolution; it first takes the form of single cells, fire up, fingering, small nests and finally, how far the tumor, the external epithelium cyst formation and stellate reticulum proliferate and neural differentiation. 
Our data support the hypothesis that ACP, create a tumor-specific cellular environment at the tumor-brain junction and probably due to the Oil Machinery Fluid content of the cysts enables neoplastic cells to quickly disperse through the adjacent tissue.

\section{Competing Interests}

The authors declare they have no conflicts of interest.

\section{Informed Consent}

Where required consent was obtained from all individual participants included in the study.

\section{Ethical Approval}

All procedures performed in studies involving human participants were in accordance with the ethical standards of the institutional and/or national research committee and with the 1964 Helsinki declaration and its later amendments or comparable ethical standards.

\section{References}

1. Louis DN, Ohgaki H, Wiestler OD, Cavenee WK, Burger PC, et al. (2007) The 2007 WHO classification of tumours of the central nervous system. Acta Neuropathol 114: 97-109.

2. Hölsken A, Buchfelder M, Fahlbusch R, Blümcke I, Buslei $R$ (2010) Tumour cell migration in adamantinomatous craniopharyngiomas is promoted by activated Wntsignalling. Acta Neuropathol 119: 631-639.

3. Kristopaitis T, Thomas C, Petruzzelli GJ, Lee JM (2000) Malignant craniopharyngioma. Arch Pathol Lab Med 124: 1356-1360.

4. Weiner HL, Wisoff JH, Rosenberg ME, Kupersmith MJ, Cohen H, et al. (1994) Craniopharyngiomas: A clinicopathological analysis of factors predictive of recurrence and functional outcome. Neurosurgery 35: 1001-1010.

5. Hunter IJ (1955) Squamous metaplasia of cells of the anterior pituitary gland. J Pathol Bacteriol 69: 141-145.

6. Ito M, Jamshidi J, Yamanaka K (2001) Does craniopharyngioma metastasize? Case report and review of the literature. Neurosurgery 48: 933-935.

7. Burghaus $S$, Hölsken A, Buchfelder M, Fahlbusch R, Riederer BM, et al. (2010) A tumor-specific cellular environment at the brain invasion border of adamantinomatous craniopharyngiomas. Virchows Arch 456: 287-300.

8. Martinez-Barbera JP, Buslei R (2015) Adamantinomatous craniopharyngioma: pathology, molecular genetics and mouse models. J Pediatr Endocrinol Metab 28: 7-17.

9. Martinez-BarberaJP(2015)60 Years of neuroendocrinology:
Biology of human craniopharyngioma: Lessons from mouse models. J Endocrinol 226: 161-172.

10. Vidal S, Kovacs K, Lloyd RV, Meyer FB, Scheithauer BW (2002) Angiogenesis in patients with craniopharyngiomas, correlation with treatment and outcomes. Cancer 94: 738745.

11. Gonzalez-Mariscal L, Betanzos A, Nava P, Jaramillo BE (2003) Tight junction proteins. Prog Biophys Mol Biol 81: $1-44$.

12. Stache C, Hölsken A, Fahlbusch R, Flitsch J, Schlaffer SM, et al. (2014) Tight junction protein claudin-1 is differentially expressed in craniopharyngioma subtypes and indicates invasive tumor growth. Neuro Oncol 16: 256-264.

13. Holsken A, Kreutzer J, Hofmann BM, Hans V, Oppel F, et al. (2009) Target gene activation of the Wnt signaling pathway in nuclear beta-catenin accumulating cells of adamantinomatous craniopharyngiomas. Brain Pathol 19: 357-364.

14. Tena-Suck ML, Morales-Del Ángel AY, Hernández-Campos ME, Fernández-Valverde F, Ortíz-Plata A, et al. (2015) Ultrastructural characterization of craniopharyngioma at the tumor boundary: A structural comparison with an experimental toxic model using "oil machinery" fluid, with emphasis on Rosenthal fibers. Acta Histochem 117: 696704.

15. Desiderio C, Martelli C, Rossetti DV, Di Rocco C, D'Angelo $L$, et al. (2013) Identification of thymosins $\beta 4$ and $\beta 10$ in paediatric craniopharyngioma cystic fluid. Childs Nerv Syst 29: 951-960.

16. Nie J, Huang GL, Deng SZ, Bao Y, Liu YW, et al. (2017) The purine receptor $P 2 X 7 R$ regulates the release of proinflammatory cytokines in human craniopharyngioma. Endocr Relat Cancer 24: 287-296.

17. Kulkarni V, Daniel RT, Pranatartiharan R (2000) Spontaneous intraventricular rupture of craniopharyngioma cyst. Surg Neurol 54: 249-253.

18. Lefranc F, Mijatovic T, Decaestecker $C$, Kaltner $H$, André $S$, et al. (2005) Monitoring the expression profiles of integrins and adhesion/growth-regulatory galectins in adamantinomatous craniopharyngiomas: Their ability to regulate tumor adhesiveness to surrounding tissue and their contribution to prognosis. Neurosurgery 56: 763-776.

19. Novak U, Kaye AH (2000) Extracellular matrix and the brain: components and function. J ClinNeurosci 7: 280-290.

20. Bhowmick NA, Moses HL (2005) Tumor-stroma interactions. Curr Opin Genet Dev 15: 97-101.

21. Andoniadou CL, Gaston-Massuet C, Reddy R, Schneider RP, Blasco MA, et al. (2012) Identification of novel pathways involved in the pathogenesis of human adamantinomatous craniopharyngioma. Acta Neuropathol 124: 259-271.

22. Müller HL (2016) Craniopharyngioma and hypothalamic injury: Latest insights into consequent eating disorders and obesity. Curr Opin Endocrinol Diabetes Obes 23: 81-89. 